\title{
Trombocitopenia trombótica inducida por vacuna. Primer reporte nacional
}

\author{
Vaccine-associated immune thrombosis and thrombocytopenia. First national report \\ Trombocitopenia trombótica induzida por vacina (VITT). Primeiro relatório nacional
}

\author{
Federico Roca ${ }^{1}$, Álvaro Danza², Antonella Pippo³ ${ }^{3}$ Ignacio Borgia ${ }^{4}$
}

\section{Resumen}

Introducción: la vacunación contra SARS-CoV-2 es una herramienta imprescindible en el combate contra la pandemia de COVID-19. La vacuna desarrollada en colaboración entre la Universidad de Oxford y el laboratorio de productos farmacéuticos AstraZeneca (AZN) ha demostrado buena eficacia, pero ha habido reporte de trombosis venosas.

Caso clínico: se presenta el caso de un paciente de 70 años, de sexo masculino, que 7 días después de la administración de la primera dosis de la vacuna AZN desarrolla trombosis venosa profunda de ambos miembros inferiores y tromboembolismo pulmonar. Coincide con trombocitopenia de $15.000 / \mathrm{mm}^{3}$, descenso del fibrinógeno y elevación de los D-dímeros. La situación clínica evoca el planteo de trombocitopenia trombótica inducida por vacuna (VITT). Se realizó tratamiento con inmunoglobulinas intravenosas, metilprednisolona y crioprecipitados. Requirió colocación de un filtro de la vena cava inferior. Una vez mejorado el recuento plaquetario se instaló tratamiento anticoagulante con apixaban. Evolucionó favorablemente.

Discusión: se trata del primer reporte nacional de VITT. Las trombosis subsiguientes a la vacuna de AZN pueden verse con las vacunas que comparten la misma plataforma vacunal (adenovirus inactivado). Se han reportado casos fundamentalmente en menores de 60 años y en topografías inhabituales. Este caso tiene la particularidad de que se trata de un paciente mayor de 60 años, que ya había tenido COVID-19 cinco meses antes y que se presenta con una trombosis en sitios habituales. El manejo terapéutico se adecuó a las pautas internacionales. El caso deja un aprendizaje relevante tanto en lo que refiere al diagnóstico precoz como al manejo terapéutico.

Palabras clave: Trombocitopenia

Trombosis

Vacuna COVID-19 AstraZeneca

Key words: $\quad$ Thrombocytopenia

Thrombosis

COVID-19 Vaccine AstraZeneca

\footnotetext{
1. Profesor Adjunto de Clínica Médica. Facultad de Medicina. Universidad de la República. Supervisor General del Departamento de Medicina y Encargado de la Unidad de Trombosis y Hemostasis de la Asociación Española Primera de Socorros Mutuos.

2. Profesor Agregado de Clínica Médica. Facultad de Medicina. Universidad de la República. Jefe del Departamento de Medicina de la Asociación Española Primera de Socorros Mutuos.

3. Internista. Coordinadora del Servicio de Emergencia de la Asociación Española Primera de Socorros Mutuos.

4. Internista e integrante de Unidad de Trombosis y Hemostasis de la Asociación Española Primera de Socorros Mutuos.

Cuenta con el consentimiento informado del paciente para la publicación.

Correspondencia: Dr. Federico Roca Errandonea. Correo electrónico: federoc@hotmail.com

Recibido: 4/6/2021

Aprobado: 28/7/2021

Attribution-NonCommercial 4.0 International (CC BY-NC 4.0)
} 


\section{Introducción}

La vacunación en el contexto actual de pandemia es la herramienta más importante para prevenir el síndrome respiratorio agudo y severo que determina la infección por SARS-CoV-2. Su efecto se basa en la disminución de la transmisibilidad y la severidad del cuadro clínico que la infección determina ${ }^{(1)}$.

Entre diciembre de 2020 y marzo de 2021 la Agencia Europea del Medicamento (EMA) aprobó la utilización de cuatro vacunas basándose en la evidencia generada en ensayos clínicos controlados y aleatorizadas. Dentro de éstas, la vacuna desarrollada en colaboración entre la Universidad de Oxford y el laboratorio de productos farmacéuticos AstraZeneca (AZN), es una vacuna con un adenovirus recombinante que expresa la glicoproteína spike del virus SARS-CoV-2. Esta vacuna ha demostrado una eficacia global de 70,4\% (IC 95\%: 54,8-80,6) en un análisis interino de un estudio aleatorizado, ciego simple de 11.636 participantes de Reino Unido y Bra$\mathrm{sil}^{(2)}$. En este estudio se identificaron 3 reacciones adversas severas asociadas a la vacuna, no siendo ninguna de ellas un evento trombótico o trombocitopenia.

En los últimos meses, la vacunación contra la COVID-19 ha avanzado a nivel mundial. La vacuna AZN se ha administrado a más de 34 millones de pacientes entre Europa y el Reino Unido, en su mayoría menores de 60 años. Desde fin de febrero de 2021 se han reportado varios casos de eventos trombóticos asociados a trombocitopenia posterior a la vacuna $\mathrm{AZN}^{(3)}$. Posteriormente se reportaron más casos en relación con la vacuna del laboratorio Janssen. Ambas vacunas emplean como plataforma vacunal a un adenovirus inactivado.

El cuadro clínico de trombosis asociada a trombocitopenia tiene características patogénicas y fisiopatológicas similares a la trombocitopenia inducida por heparina (HIT), la cual corresponde a una trombocitopenia inmune vinculada a la unión de anticuerpos de tipo inmunoglobulina $\mathrm{G}$ a la superficie plaquetaria, denominados anticuerpos anti-PF4. Este síndrome se ha denominado trombocitopenia trombótica inducida por vacuna (VITT) o síndrome de trombocitopenia y trombosis relacionada con vacuna, TTS por su acrónimo en inglés. Desde su descripción y hasta el 7 de abril de 2021 se han reportado 79 casos, predominando en pacientes de sexo femenino, menores de 60 años, siendo la trombosis venosa cerebral la forma de presentación clínica más frecuente. Le siguen en frecuencia la trombosis venosa esplácnica y finalmente el tromboembolismo pulmonar $(\mathrm{TEP})^{(4,5)}$.

En Uruguay la campaña de vacunación ha avanzado en forma acelerada desde marzo de 2021. Se ha realizado en base a la administración de las vacunas de los labo- ratorios Pfizer/BionTech, Sinovac y AZN. En virtud de las alertas emitidas por diversos organismos regulatorios, la autoridad sanitaria determinó que esta última se administrara a la población mayor a 60 años. Hasta el momento se han administrado 81 mil dosis de la vacuna de AZN.

El siguiente reporte describe y analiza el primer caso clínico compatible con trombocitopenia trombótica inducida por la vacuna AZN en Uruguay. A los efectos de presentar este reporte se ha obtenido autorización de la institución de salud en donde el caso ha sido asistido y se procedió a recabar y obtener el consentimiento informado del usuario, bajo compromiso de utilizar los datos de forma anónima. El caso fue reportado a la autoridad sanitaria nacional en materia de farmacovigilancia.

\section{Caso clínico}

\section{Presentación clínica}

Paciente de 77 años, sexo masculino, sin antecedentes patológicos ni consumo de fármacos o tóxicos. Cursó infección leve por COVID-19 en enero de 2021 confirmada por PCR para SARS-CoV-2. No requirió internación y se otorgó alta al día 14 de inicio de síntomas sin evidencia de complicaciones. No recibió tromboprofilaxis farmacológica durante la enfermedad. Luego de 3 meses del alta epidemiológica y 7 días posteriores a la primera dosis de la vacuna AZN, comienza con edema de ambos miembros inferiores hasta raíz de muslo. En la evolución agrega dolor torácico y expectoración hemática. No refiere otros sangrados. Niega otros síntomas respiratorios.

Consulta en el servicio de emergencia donde se confirma trombosis venosa profunda (TVP) bilateral proximal, con compromiso de vena femoral común, superficial y poplítea, por ecografía Doppler de miembros inferiores. Se confirma el diagnóstico de TEP bilateral multisegmentario por angiotomografía computarizada de tórax. De la analítica se destaca: plaquetopenia de $15.000 / \mathrm{mm}^{3}$ (valores de referencia: $130.000-400.000 / \mathrm{mm}^{3}$ ) confirmada con lámina periférica, ausencia de esquistocitos, sin otras citopenias. Fibrinógeno descendido $(120 \mathrm{mg} / \mathrm{dl}$; valor de referencia: $200-400 \mathrm{mg} / \mathrm{dl}$ ), TP $67 \%$ (valor de referencia: 70\%-120\%), aPTT 25 segundos (valor de referencia: 24-36 segundos); D-dímeros elevados ( $>20.000$ $\mathrm{ng} / \mathrm{ml}$; valor de referencia $<500 \mathrm{ng} / \mathrm{ml}$ ).

Se realizó una tomografía axial computarizada de tórax, abdomen y pelvis sin evidencia de tumoraciones ni adenomegalias, así como angiotomografía de abdomen, que no evidenció trombosis en la circulación arterial ni venosa abdominal. El paciente no presentó síntomas neurológicos, el examen neurológico, incluyendo el fondo de ojo, fue normal. Las serologías para los virus 


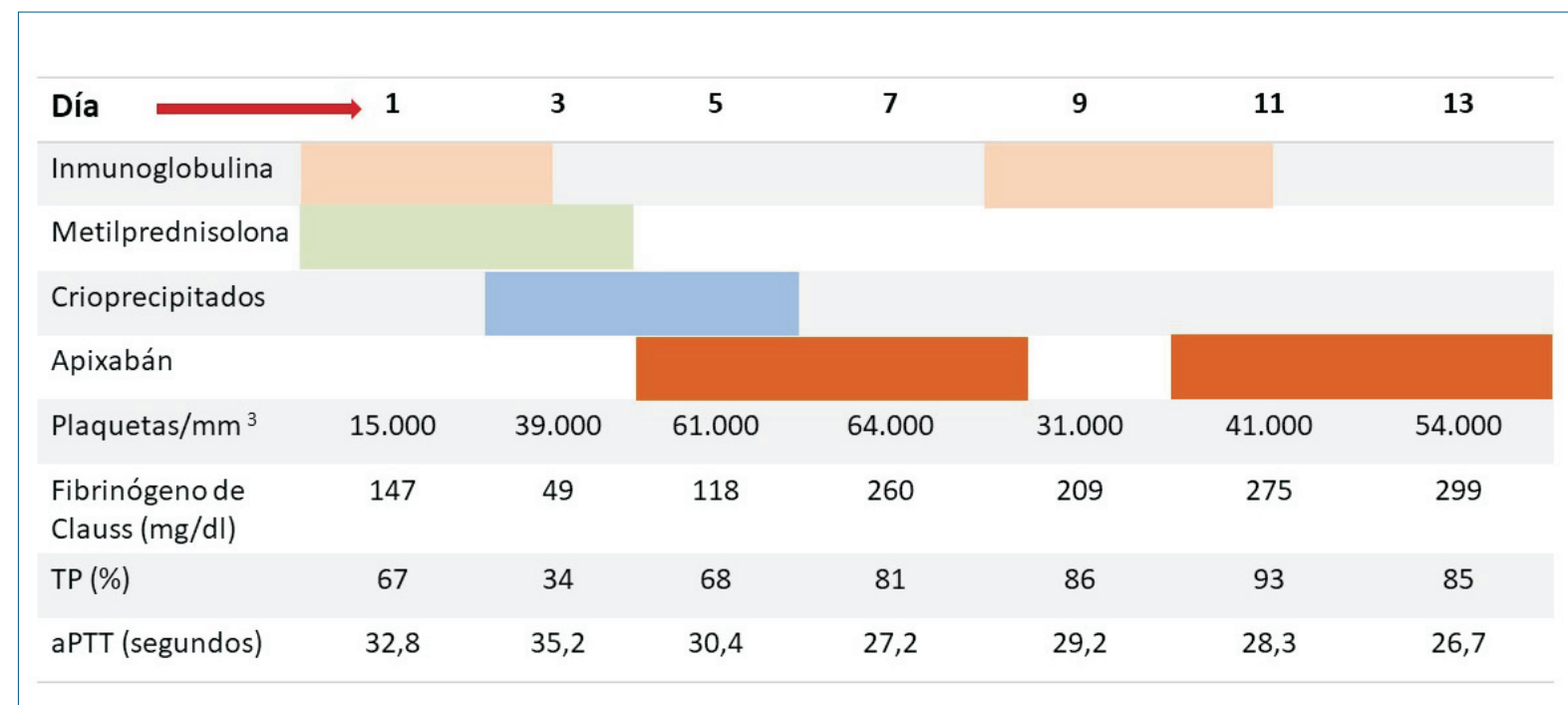

Figura 1. Tratamientos administrados y evolución de los parámetros analíticos más relevantes al caso clínico. Nota: TP: tasa de protombina. aPTT: tiempo de tromboplastina parcial activada.

de la hepatitis B, C y HIV fueron negativas. El hepatograma y la función renal fueron normales.

\section{Manejo terapéutico}

Con planteo de VITT, se administró inmunoglobulina intravenosa a dosis de $0,4 \mathrm{~g} / \mathrm{kg}$ en dos dosis, metilprednisolona intravenosa $500 \mathrm{mg} /$ día por 3 días desde el día del ingreso. Dada la trombocitopenia severa que contraindicaba el uso de anticoagulantes, se colocó filtro de la vena cava inferior (removible) para prevenir la recurrencia del TEP. Presentó como complicación un hematoma extenso inguinal derecho, en relación con el sitio de punción para emplazamiento del filtro cava, que en la evolución determinó una caída de la hemoglobina a $8,8 \mathrm{~g} / \mathrm{dl}$ (valor de referencia para sexo masculino: $13-18 \mathrm{~g} / \mathrm{dl})$.

Con respecto a la evolución del recuento plaquetario, fue en ascenso hasta el día 7 de la administración de inmunoglobulina intravenosa, logrando un recuento mayor a $50.000 / \mathrm{mm}^{3}$ al $5^{\circ}$ día de inicio del tratamiento (figura 1). Posteriormente se constata un nuevo descenso progresivo por lo que se procedió a administrar una nueva dosis de $0,4 \mathrm{~g} / \mathrm{kg}$ de inmunoglobulina intravenosa, en dos dosis.

Con respecto a las alteraciones de la crasis, empeoraron en los primeros 5 días, lo cual se vinculó al consumo de factores por trombosis mantenida y por el extenso hematoma en el sitio de punción del filtro cava. Requirió reposición con crioprecipitados en 3 oportunidades, logrando corregir dicho trastorno en forma definitiva.

Se inició anticoagulación con apixaban luego de 5 días de inicio de tratamiento y luego de obtener un re- cuento plaquetario por encima de $50.000 / \mathrm{mm}^{3}$ y un fibrinógeno de Clauss mayor a $100 \mathrm{mg} / \mathrm{dl}$.

Al mes se retiró el filtro de la vena cava inferior sin complicaciones, actualmente presenta recuento plaquetario de $90.000 / \mathrm{mm}^{3}$, crasis sanguínea en rangos normales y está recibiendo apixaban $5 \mathrm{mg}$ cada 12 horas por vía oral.

\section{Discusión}

La presentación clínica y la ausencia de diagnósticos diferenciales permiten concluir que efectivamente se trata de un caso de VITT. Los criterios diagnósticos (tabla 1) incluyen la dosificación de anticuerpos anti-PF4, siendo éstos últimos necesarios para definirlo como caso confirmado. Actualmente en Uruguay no está disponible la dosificación de estos anticuerpos. Por este motivo se realiza un diagnóstico de presunción en base a los siguientes aspectos: presencia de eventos trombóticos en un plazo de 4 a 20 días posteriores a la administración de la primera dosis de la vacuna AZN, descenso del recuento plaquetario por debajo de 20 mil plaquetas $/ \mathrm{mm}^{3}$, elevación significativa de los D-dímeros y descenso del fibrinógeno. En consecuencia, si bien el diagnóstico no puede confirmarse por la ausencia de los anticuerpos específicos, se realiza diagnostico con elevado nivel de certeza.

Sin perjuicio del diagnóstico de VITT, el caso clínico tiene al menos tres particularidades llamativas. La primera es la edad de presentación clínica. En efecto, es de destacar que hasta marzo-abril de 2021 la vacuna AZN estaba aprobada y se administraba a los menores de 60 años. Este hecho podría estar subestimando la in- 
Tabla 1. Criterios de presunción y de confirmación diagnóstica de trombocitopenia trombótica por vacuna (VITT).

\begin{tabular}{|c|c|}
\hline $\begin{array}{l}\text { Elementos de presunción clínica } \\
\qquad y \text { analítica }\end{array}$ & Confirmación diagnóstica \\
\hline $\begin{array}{l}\text { Secuencia temporal: } 4 \text { a } 20 \text { días } \\
\text { post-vacunación (mayor } \\
\text { frecuencia en primera dosis) } \\
\text { Trombosis confirmadas por } \\
\text { estudios de imagen } \\
\text { Trombocitopenia confirmada por } \\
\text { lámina periférica } \\
\text { D-dímeros elevados } \\
\text { Fibrinógeno normal o descendido }\end{array}$ & $\begin{array}{l}\text { Anticuerpos contra el } \\
\text { complejo PF4 }\end{array}$ \\
\hline
\end{tabular}

cidencia de este síndrome en mayores de 60 años. Posterior a los reportes de VITT, y en base a recomendaciones de la EMA, comenzó a utilizarse únicamente en mayores de 60 años. Por este motivo, sería al menos probable que su incidencia en mayores de 60 años aumente progresivamente a medida que aumenta la vacunación en esa franja etaria con la vacuna AZN. La segunda característica distintiva es el antecedente de infección por COVID-19. Los casos clínicos descritos previamente no hacen referencia al antecedente de infección previo a la vacunación. Sería interesante poder identificar si la infección previa constituye un factor de riesgo para desarrollar VITT o si es una simple asociación producto del azar. La tercera, es la presentación clínica con TVP/TEP, que no suele ser lo más frecuente. Esta particularidad podría ser fortuita, pero también podría estar en relación con la franja etaria que se encuentra siendo vacunada actualmente con esta vacuna.

No ha habido hasta el mes de mayo de 2021 casos reportados de pacientes que requirieran de colocación de filtro de la vena cava inferior en el curso de un VITT. El procedimiento se realizó con éxito y se planificó y concretó su remoción luego de lograr la anticoagulación a dosis plenas, corrección de crasis y elevación de plaquetas por encima de $50.000 / \mathrm{mm}^{3}$. De hecho, se optó por la colocación de un filtro removible, para evitar las complicaciones a largo plazo que estos implantes puedieran determinar.

En el caso clínico presentado hay una secuencia temporal clara entre la administración de la vacuna $\mathrm{AZN}$ y el evento trombótico. Sin perjuicio de ello, es razonable plantear como diagnósticos diferenciales la coagulación intravascular diseminada (CID) aguda y el púrpura trombocitopénico trombótico (PTT). En relación con el primer planteo, en el caso reportado no existen elemen- tos favorecedores, como infecciones graves, enfermedades neoplásicas ni traumatismos significativos. Además, la presentación más frecuente en la CID es el sangrado respecto a las manifestaciones trombóticas, el recuento plaquetario no suele ser tan bajo como en nuestro caso; a esto se suma el rápido ascenso plaquetario con el tratamiento con inmunoglobulina, todo lo cual permite alejar este planteo ${ }^{(6)}$. En relación con el PTT, el paciente tenía crasis alterada y ausencia de esquistocitos en la lámina periférica, lo cual está en contra de este planteo.

Dado que se trata de un paciente de 70 años, es razonable realizar un tamizaje de neoplasias ocultas, como fue realizado en este caso. Se optó por un tamizaje basado en estudios de imagen, dosificación de antígeno prostático específico, búsqueda de pérdidas sanguíneas intestinales, dosificación de calcio sérico, estudio del hepatograma y de la función renal, examen de orina y proteinograma electroforético. La normalidad de esos estudios contribuye a apoyar el planteo realizado.

Por otra parte, la duración del tratamiento anticoagulante es incierta, no existiendo estudios prospectivos que sirvan de referencia para tomar una decisión. En principio, parece razonable que una vez superado el factor favorecedor del evento trombótico -en este caso la vacunación- se pueda plantear la suspensión del tratamiento anticoagulante. Esto no deberá ser antes de los 3 meses posteriores a la normalización del recuento plaquetario. Algunos pacientes tendrían un riesgo mayor de recurrencia, como son los que persisten positivos para los anti-PF4, en cuyo caso la decisión será más compleja. En cualquier caso, es razonable realizar dosificación de plaquetas y D-dímeros para control evolutivo de estos pacientes ${ }^{(7,8)}$.

Si bien la eficacia y seguridad de la vacuna AZN ha sido demostrada, el número de pacientes mayores de 70 años incluidos en los ensayos clínicos pivotales es bajo. El estudio que permitió su aprobación inicial por parte de la EMA incluyó menos de $10 \%$ de pacientes en esa franja etaria en un total de 14.000. Un estudio más reciente incluyó 260 pacientes mayores de 70 años ${ }^{(9)}$. Considerando la incidencia planteada de este síndrome, parece necesaria la realización de estudios con mayor cantidad de pacientes para poder establecer con más claridad la incidencia y las características de este cuadro clínico en los pacientes mayores de 60 años.

Es necesario destacar que los pacientes con factores de riesgo protrombóticos o con antecedentes de haber tenido trombosis venosa no tienen contraindicación para recibir la vacuna AZN ni otras vacunas que compartan la misma plataforma vacunal ${ }^{(10)}$. En efecto, debe tenerse en cuenta que se trata de una complicación poco frecuente por lo cual parece claro que los beneficios superan ampliamente los riesgos. 
Finalmente, parece importante hacer referencia a la conducta a tomar con respecto a la inmunización en los pacientes que cursaron un episodio de VITT frente la primera dosis de la vacuna. El Centro de Control de Enfermedades de Estados Unidos de América (CDC) realiza una recomendación que se basa en completar la inmunización con una vacuna cuya plataforma vacunal sea diferente, concretamente con ARN mensajero, como las vacunas de los laboratorios Pfizer/BionTech o de Moderna, en el correr de los 90 días siguientes ${ }^{(10)}$. Esta recomendación es intuitiva, puesto que no existe evidencia sólida que la sustente. Parece claro que se debe contraindicar la posterior inmunización con una vacuna que comparta el vector con AZN.

\section{Conclusiones}

Considerando la disponibilidad de vacuna AZN en nuestro medio, la incidencia estimada de VITT en la población vacunada y la morbimortalidad del cuadro clínico, parece necesario acceder a la de detección de anticuerpos plaquetarios anti-PF4 con el objetivo de poder confirmar los diagnósticos en forma oportuna. Además, es de destacar que la persistencia de positividad de estos anticuerpos podría tener implicancias en la duración del tratamiento anticoagulante, reforzando su utilidad.

Es probable que la presentación clínica de este cuadro pueda ser distinta en los pacientes mayores de 60 años. Es necesario estar alerta frente a la aparición de más casos en esta franja etaria e insistir en su reporte.

La utilización del filtro de la vena cava parece una alternativa útil en las complejas situaciones en las que existe trombosis venosa de los miembros inferiores y trombocitopenia severa que contraindique el uso de anticoagulantes en agudo.

Son necesarios estudios en vida real con mayor cantidad de pacientes para poder estimar con mayor certeza la incidencia, características clínicas y factores de riesgo de esta nueva entidad.

En las guías de práctica clínica es necesario abordar los puntos de duración del tratamiento anticoagulante y las recomendaciones con respecto a la inmunización subsiguiente de los pacientes que han presentado VITT.

\section{Abstract}

Introduction: vaccines against SARS-CoV-2 are an essential tool against the COVID-19 pandemic. The vaccine developed in collaboration with the University of Oxford and the AstraZeneca (AZN) laboratory has proved to be effective, although venous thrombosis have been reported.

Clinical case: the study presents the case of a 70 year old male patient who, 7 days after receiving the first dose of the AZN vaccination develops deep vein throm- bosis (DVT) in the lower extremities and pulmonary embolism.

Simultaneously, thrombocytopenia is $15.000 / \mathrm{mm}^{3}$, fibrinogen levels drop D-dimer levels are elevated. The clinical situation leads to the suspicion of vaccine-associated immune thrombosis and thrombocytopenia (VITT). The patient was treated with intravenous immune globulin, methylprednisolone and cryoprecipitates, requiring a filter to be placed in the inferior vena cava. Once platelets count improved, anti-coagulation therapy including apixaban was commenced, evolution being good.

Discussion: this is the first national report on VITT. Thrombosis after the AZN vaccination may be seen in other vaccines that use the same vaccine platform (inactive adenovirus). Cases have been reported mainly in patients younger than 60 years old and in unusual topographies. In particular, this case presents a male patient that is older than 60 years old, who had already been infected with COVID-19 five months before and who currently consults with thrombosis in regular sites. Therapeutic handling observed international guidelines. The case contributes relevant data both in terms of early diagnosis and therapeutic handling.

\section{Resumo}

Introdução: a vacinação contra a SARS-CoV-2 é uma ferramenta essencial na luta contra a pandemia de COVID-19. A vacina desenvolvida pela colaboração entre a Universidade de Oxford e o laboratório farmacêutico AstraZeneca (AZN) tem demonstrado boa eficácia, mas foram relatados casos de trombose venosa.

Caso clínico: apresenta-se o caso de um paciente do sexo masculino, 70 anos, que 7 dias após a administração da primeira dose da vacina AZN desenvolveu trombose venosa profunda de ambos os membros inferiores $\mathrm{e}$ tromboembolismo pulmonar. Coincide com trombocitopenia de $15.000 / \mathrm{mm} 3$, diminuição do fibrinogênio e aumento dos D-dímeros. A situação clínica lembra a trombocitopenia trombótica induzida por vacina (VITT). O tratamento foi realizado com imunoglobulinas intravenosas, metilprednisolona e crioprecipitados. Foi necessário colocar um filtro de veia cava inferior. Uma vez que a contagem de plaquetas melhorou, o tratamento anticoagulante com apixaban foi instalado. $\mathrm{O}$ paciente favoravelmente.

Discussão: este é o primeiro relatório nacional de VITT. As tromboses subseqüentes à vacina $\mathrm{AZN}$ podem ser vistas com vacinas que compartilham a mesma plataforma (adenovírus inativado). Os casos foram relatados principalmente em pessoas com menos de 60 anos de idade e em topografias incomuns. Este caso tem a particularidade de se tratar de um paciente com mais de 60 
anos, já com COVID-19 há cinco meses e que apresenta trombose em sítios comuns. O manejo terapêutico foi adaptado às diretrizes internacionais. $\mathrm{O}$ caso deixa um aprendizado relevante tanto no que diz respeito ao diagnóstico precoce quanto ao manejo terapêutico.

\section{Bibliografía}

1. World Health Organization. Draft landscape of COVID-19 candidate vaccines. Disponible en: https://www.who.int/publications/m/item/draft-landscape-of-covid-19-candidate-vaccines [Consulta: 1 junio 2021].

2. Voysey M, Clemens SAC, Madhi SA, Weckx LY, Folegatti PM, Aley PK, et al. Safety and efficacy of the ChAdOx1 nCoV-19 vaccine (AZD1222) against SARS-CoV-2: an interim analysis of four randomised controlled trials in Brazil, South Africa, and the UK. Lancet 2021; 397(10269):9-111.

3. European Medicines Agency. COVID-19 Vaccine AstraZeneca: benefits still outweigh the risks despite possible link to rare blood clots with low blood platelets. Amsterdam, March 2021.

4. Franchini M, Liumbruno GM, Pezzo M. COVID-19 Vaccine-associated Immune thrombosis and thrombocytopenia (VITT): diagnostic and therapeutic recommendations for a new syndrome. Eur J Haematol 2021; 107(2):173-80.

5. Danza Á, Frantchez V. Sobre la vacuna desarrollada en Reino Unido (Universidad de Oxford-AstraZeneca). Rev Méd Urug 2021; 37(2):e37215.
6. Levi M, Toh CH, Thachil J, Watson HG. Guidelines for the diagnosis and management of disseminated intravascular coagulation. British Committee for Standards in Haematology. Br J Haematol 2009; 145(1):24-33.

7. Greinacher A, Thiele T, Warkentin TE, Weisser K, Kyrle P, Eichinger S. A prothrombotic thrombocytopenic disorder resembling heparin-induced thrombocytopenia following coronavirus-19 vaccination. Research Square Preprint. doi: 10.21203/rs.3.rs-362354/v1.

8. Pavord S, Lester W, Makris M, Scully M, Hunt B. Guidance from the Expert Haematology Panel (EHP) on Covid-19 Vaccine-induced Immune Thrombocytopenia and Thrombosis (VITT). London: British Society for Haematology, 2021.

9. Ramasamy MN, Minassian AM, Ewer KJ, Flaxman AL, Folegatti PM, Owens DR, et al. Safety and immunogenicity of ChAdOx $1 \mathrm{nCoV}-19$ vaccine administered in a prime-boost regimen in young and old adults (COV002): a single-blind, randomised, controlled, phase $2 / 3$ trial. Lancet 2021; 396(10267):1979-93.

10. Centers for Diseases and Prevention. Interim clinical considerations for use of mRNA COVID-19 vaccines currently authorized in the United States. CDC, 2021. Disponible en: https://www.cdc.gov/vaccines/covid-19/info-by-product/clinical-considerations.html [Consulta: 1 junio 2021].

\section{Contribución de autores}

Federico Roca, ORCID 0000-0002-8666-4056. Concepción, diseño, análisis del caso clínico e interpretación de los resultados, redacción del manuscrito y revisión final.

Álvaro Danza, ORCID 0000-0001-9070-2230. Concepción, interpretación de resultados, redacción del manuscrito y revisión final.

Antonella Pippo, ORCID 0000-0003-1690-3629. Concepción y revisión final.

Ignacio Borgia, ORCID 0000-0002-8562-0481. Concepción y revisión final. 